\title{
Low Illumination Image Enhancement based on Improved Retinex Algorithm
}

\author{
Yuan-Bin Wang*, Qian Han, Yu-Jie Li, Yuan-Yuan Li \\ School of Electrical and Control Engineering, Xi'an University of Science and Technology, Yanta Road 58, Xi'an 710054, \\ China \\ 13379232752@163.com,947674382@qq.com,1678931975@qq.com,2764845392@qq.com \\ Received 22 April 2021; Revised 28 August 2021; Accepted 6 October 2021
}

\begin{abstract}
Aiming at the problems of insufficient illumination and low contrast of low illumination image, an improved Retinex low illumination image enhancement algorithm is proposed. Firstly, the brightness component $\mathrm{V}$ of the original image is extracted in HSV color space, and its enhancement by Single-Scale Retinex (SSR) is used to obtain the reflection component. For the edge problem caused by the estimation of illumination component, the Gaussian weighted bilateral filter is used as the filter function to maintain the edge information. Then, the saturation component $\mathrm{S}$ is adaptively stretched to improve the color saturation. However, different low illumination images have different contrast, and some images have insufficient contrast enhancement, so a global adaptive algorithm is introduced to modify the contrast and obtain the final image. According to the logarithmic characteristics of human vision, it can adaptively enhance the contrast of different images without over enhancement. Experimental results show that the proposed algorithm can effectively improve the visual quality of the image, the contrast is improved significantly and image edge details are protected, and objective evaluations such as average gradient, information entropy and peak signal-to-noise ratio have been improved.
\end{abstract}

Keywords: low illumination image, Retinex, image enhancement, bilateral filtering, logarithmic enhancement

\section{Introduction}

Under the condition of night or backlight, the captured image has problems such as poor brightness and contrast, which affects the visual effect of human eyes on the image and subsequent analysis, such as information extraction and target recognition. Using low illumination image enhancement technology can reduce the impact of low illumination environment on the image, enhance the scene characteristics of the image, and achieve effective information visualization effect. Therefore, low illumination image enhancement algorithm has important research significance [1-2].

Low illuminance image enhancement is mainly to improve image contrast and suppress dark area. At present, low illumination image enhancement is mainly based on spatial domain, frequency domain and deep learning methods.

Spatial enhancement algorithms include histogram equalization, Retinex algorithm and so on. Histogram equalization algorithm stretches the gray range to the uniformly distributed gray range, which effectively improves the image contrast, but it is prone to color difference and will lead to loss of detail information [3-4]. Dong Lili et al. [5] proposed the improved histogram HEEF algorithm to protect image details and improve information entropy, but there will be over enhancement phenomenon. The Retinex algorithm was proposed by land [6] in the 1970s, and then derived Single-Scale Retinex (SSR) algorithm, Multi-Scale Retinex (MSR) algorithm, MultiScale Retinex (MSRCR) algorithm with color restoration [7-8]. However, these methods generally exist halo phenomenon at the edge of the image with drastic pixel changes, and are accompanied by color distortion. Therefore, a large number of Retinex improvements continue to appear. Among them, Kimmel [9] et al. proposed a variation based Retinex image enhancement method, which has greatly improved the processing effect compared with the traditional Retinex method. Mou Qi et al. [10] used guided filtering to deal with problems such as blurred edge contour and loss of details caused by MSR, but it was prone to be affected by uneven illumination and partial color distortion occurred after enhancement.

Wavelet transform based on frequency domain enhancement algorithm is widely used, which mainly decomposes the image into low-pass sub-image and high-pass sub-image. The low-pass sub-image represents low-frequency information and corresponds to the image smoothing region. The high-pass sub-image represents the image high-frequency information and corresponds to the image detail information. Then, different frequency coefficients are enhanced through various enhancement algorithms to improve the image contrast and highlight 
the image detail information. Fu Zhengfang et al. [11] proposed a low illumination image enhancement algorithm based on wavelet transform, which used different enhancement coefficients for hierarchical processing of different frequency characteristics to realize image enhancement while preserving scene details. However, the selection of wavelet base needs to be defined in advance, and the real-time performance of the algorithm is limited. Li Qingzhong et al. [12] proposed an adaptive enhancement algorithm for low illumination images based on wavelet transform, which made use of histogram objective function and simulated annealing optimization algorithm, it has good real-time performance but insufficient visual effect.

In recent years, deep learning algorithm has also been applied in the field of image enhancement. It mainly extracts image features through convolutional neural network to obtain the mapping from low illumination images to clear images. Li et al. [13] trained the deep convolution neural network (LightenNet) to perform the low light image enhancement task. Ma Hongqiang et al. [14] used the deep convolution neural network method to extract features and conduct sample training, which effectively improved the brightness and contrast and avoided color distortion. However, the network model needs a lot of data and the training time is long, and the lack of theoretical basis makes the network design a challenge.

In view of the shortcomings of the above algorithms, a low illumination image enhancement algorithm based on improved Retinex is proposed in this paper. The edge details are protected by bilateral filtering, and then the global adaptive algorithm is introduced for contrast correction. Experimental results show that this method can effectively improve the contrast and brightness of all kinds of low illumination images, and there will be no local too bright or too dark and halo phenomenon.

\section{Improved Retinex Algorithm}

\subsection{Retinex Principle}

In Retinex theory, the color of an object is determined by the reflection ability of long wave, medium wave and short wave, rather than by the absolute intensity of reflected light, and the color of an object is not affected by illumination heterogeneity. The image observed by human eyes depends on the reflection of incident light on the surface of the object [15-16]. An image $I(x, y)$ can be decomposed into the illumination component $L(x, y)$ of incident light and the reflection component $R(x, y)$ of the target object, which can be expressed by the formula:

$$
I(x, y)=R(x, y) * L(x, y)
$$

Then take the logarithm on both sides, expressed as

$$
\log (R(x, y))=\log (I(x, y))-\log (L(x, y))
$$

In SSR algorithm, calculating the illumination component of incident light is an uncertain mathematical problem, which can only be solved by approximation [17]. Considering the transformation degree of illumination is slow and belongs to low-frequency components, the SSR generally uses the low-pass filter function as the central surround function to estimate the illumination component. The expression of image incident light is:

$$
L(x, y)=I(x, y) * F(x, y)
$$

The Gaussian filter function $f(x, y)$ is expressed as

$$
F(x, y)=\frac{1}{2 \pi \sigma^{2}} \exp \left(-\frac{x^{2}+y^{2}}{2 \sigma^{2}}\right)
$$

Substitute equation (3) into equation (2), and we can obtain 


$$
\log (R(x, y))=\log (I(x, y)-\log (I(x, y) * F(x, y)))
$$

Finally, an exponential transformation is performed to obtain the reflection $R(x, y)$ of the image, which is enhanced by the Retinex algorithm.

\subsection{Limitations of the Retinex Algorithm}

Traditional Retinex algorithm assumes that the illumination component is uniformly distributed, so the Gaussian low-pass filter is employed to estimate the illumination of the image. In the actual natural environment, the light distribution is not uniform, the Gaussian low-pass filter will lead to low estimation of the dark region and high estimation of the bright region, which is very easy to appear halo phenomenon and has a negative impact on the subsequent image processing. The low illumination image is enhanced by SSR and shown in Fig. 1.
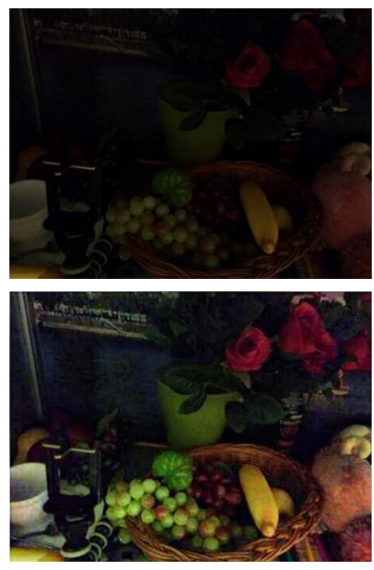

a
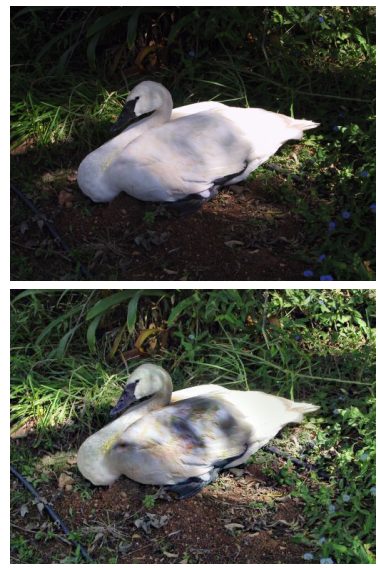

b
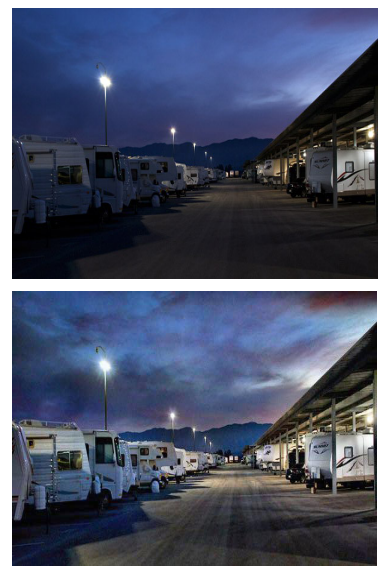

$\mathrm{c}$
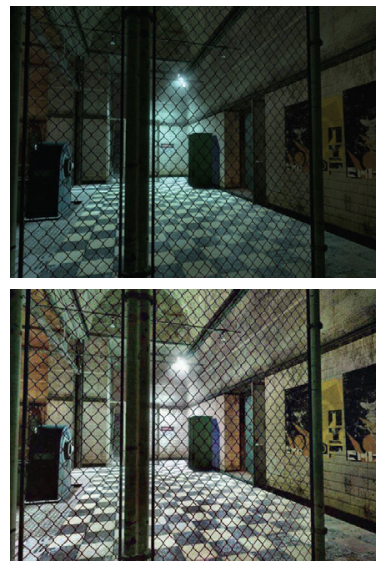

d

Fig 1. Traditional Retinex enhancement results

Compared with Fig. 1, it can be found that the contrast is significantly improved, but the halo phenomenon occurred nearby the light and the contrast is not very good, because when the low illumination image is processed, the illumination is an underdetermined problem in mathematics, which can only be obtained by approximate estimation. Considering that the slow change of light, the intensity of the pixels at the edges of the image will affect the low image contrast. Therefore, the halo phenomenon and the problem of insufficient contrast should be improved.

\section{Improved Retinex Algorithm}

Aiming at the limitation of SSR, this paper proposes an improved Retinex algorithm. The algorithm includes four parts: Firstly, the gaussian weighted bilateral filter is employed to estimate the illumination of the original image, which can avoid the halo phenomenon and keep the image edge effectively; Secondly, in the HSV color space, the brightness component $\mathrm{V}$ and the saturation component $\mathrm{S}$ are enhanced by the SSR and adaptive nonlinear stretching, respectively, then an enhanced image with better reflection components at the edges and saturation is obtained; Thirdly, the reflection component is exponential to get the enhanced luminance component, and then convert the HSV color space to RGB space; Finally, the global adaptive algorithm is introduced for contrast correction to obtain the final enhanced image. The working flowchart is shown in Fig. 2:

\subsection{Saturation Component Adaptive Nonlinear Stretching}

In order to make the color of the image fuller, we need to stretch the saturation. There are three color channels of $R, G$, and $B$ in the color image. When Retinex enhancement is carried out, the three channels should be enhanced, respectively. However, the three components in the RGB color space have a strong correlation, and enhances 
them will destroy the correlation and lead to image color distortion. In the HSV color space, the hue, saturation, and brightness components can be separated, and the original color structure of the image can be better retained while improving the brightness and saturation of the image. In this paper, RGB space is transferred to HSV space, where $T_{\max }$ is the maximum value in $R, G$, and $B$, and $T_{\max }$ is the minimum value. The relation is shown in equation (6).

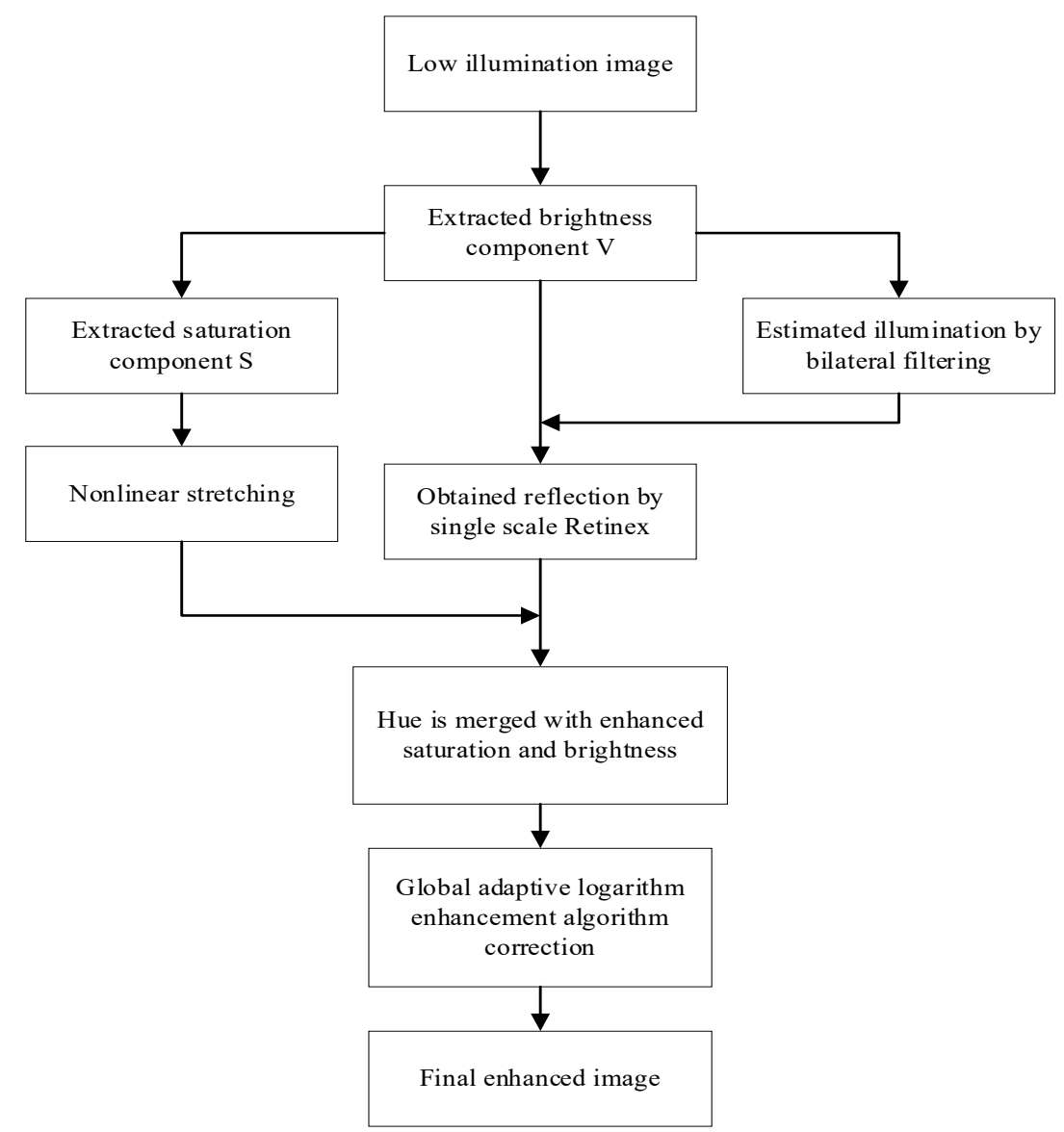

Fig. 2. Working flow chart of improved Retinex algorithm

$$
\begin{aligned}
& \left\{\begin{array}{l}
S=\left\{\begin{array}{c}
0, \text { if } T_{\text {max }}=0 \\
\frac{T_{\text {max }}-T_{\min }}{T_{\text {max }}}, \text { otherwise }
\end{array}\right. \\
H^{\prime}=\left\{\begin{array}{c}
60 \times \frac{G-B}{T_{\text {max }}-T_{\text {min }}}, T_{\text {max }}=R \\
120+60 \times \frac{B-R}{T_{\text {max }}-T_{\text {min }}}, T_{\text {max }}=R \\
240+60 \times \frac{R-G}{T_{\text {max }}-T_{\text {min }}}, T_{\text {max }}=R
\end{array}\right.
\end{array}\right. \\
& V=T_{\max } \\
& H=\left\{\begin{array}{c}
H^{\prime} \quad, \text { if } H^{\prime} \geq 0 \\
H^{\prime}+360^{\circ}, \text { otherwise }
\end{array}\right.
\end{aligned}
$$


Because the saturation of different low illuminance images is different, the saturation component is extracted after the image is converted to HSV color space, then an adaptive nonlinear stretching algorithm is employed, expressed as

$$
\mathrm{S}_{\text {out }}=\left[1+\frac{\operatorname{mean}(R, G, B)}{\max (R, G, B)+\min (R, G, B)+1}\right]
$$

Where $S_{\text {in }}$ and $S_{\text {out }}$ are the saturation before and after stretching, respectively, $\operatorname{mean}(R, G, B)$, $\max (R, G, B)$, and $\min (R, G, B)$ are the averages, maximum and minimum values of the color components of $R, G$ and $B$ corresponding to the image.

\subsection{Bilateral Filter}

Using bilateral filtering as filtering function can maintain image edge and avoid halo phenomenon [18-20]. Since the Gaussian weighted average is adopted in the bilateral filter, it is divided into the Gaussian space weight and the Gaussian similar weight. The gaussian space weight is related to the pixel position, which is the Euclidean distance of the pixel, and the Gaussian similarity weight is related to the size of pixel value, which is the similarity of the pixel value. The bilateral filter formula is defined as

$$
I(x, y)=\frac{\sum_{(k, l) \in S(i, j)} f(k, l) w(i, j, k, l)}{\sum_{(k, l) \in S(i, j)} w(i, j, k, l)} .
$$

Where $I(x, y)$ is the output image, and $S(i, j)$ is a rectangular sliding window centered on $(i, j), f(k, l)$ is the sliding window area of the input image, and $w(i, j, k, l)$ is the kernel function of Gaussian space weight and similar weight. The kernel function is defined as

$$
w(i, j, k, l)=\exp \left(-\frac{(i-k)^{2}+(j-l)^{2}}{2 \sigma_{d}^{2}}-\frac{\|f(x, y)-f(k, l)\|^{2}}{2 \sigma_{r}^{2}}\right)
$$

$\sigma_{d}, \sigma_{r}$ are the variances of spatial domain and similarity domain, respectively.

In the flat area of the image, the pixel value of the image changes in small magnitude, and the space weight plays a significant role, which is equivalent to gaussian blur. In the edge area of the image, the variation range of pixel value is large, and the similarity weight becomes large, so edge information is maintained. Therefore, the bilateral filter can be used to adaptively estimate the difference between the edges of low illumination images. It can keep the edge information of the image and avoid the halo phenomenon effectively.

\section{Global Adaptive Algorithm Correction}

The contrast enhancement degree of different images is different. When the low illumination image is enhanced by Retinex, the image needs to be corrected. From Weber's law, the perceived brightness of the human visual system is approximately logarithmic function [21]. The logarithmic transform based on the spatial domain can improve the contrast of the dark areas and enhance the image details of the dark part. According to the tone mapping relationship in reference [22], a global adaptive logarithmic enhancement algorithm is employed to perform contrast correction, expressed as

$$
L_{g}(x, y)=\frac{\log \left(L_{w}(x, y) / \overline{L_{w}}+1\right)}{\log \left(L_{w \max } / \overline{L_{w}}+1\right)} .
$$


Where $L_{g}(x, y)$ is the result of global adaptive output, $L_{w}(x, y)$ is the brightness value of the input image, $L_{w \max }, \overline{L_{w}}$ are the maximum and logarithm mean of the brightness of the input image, respectively, expressed as

$$
\overline{L_{w}}=\exp \left(\frac{1}{N} \sum_{x, y} \log \left(\delta+L_{w}(x, y)\right)\right)
$$

Where $\mathrm{N}$ is the total number of pixels, and $\delta$ is a smaller value to avoid overflow during logarithmic calculation. Make a global adaptive logarithmic enhancement output curve, as shown in Fig. 3(a), Fig. 3(b), Fig. 3(c).

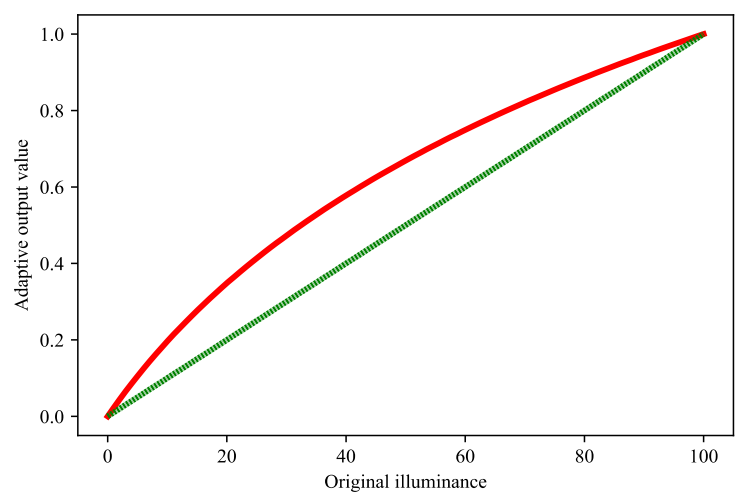

(a) Logarithmic mean 30, brightness 100

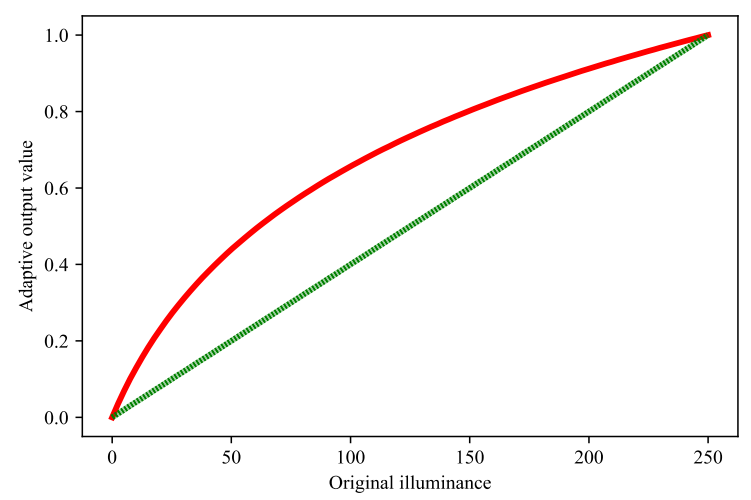

(b) Logarithmic mean 30, brightness 250

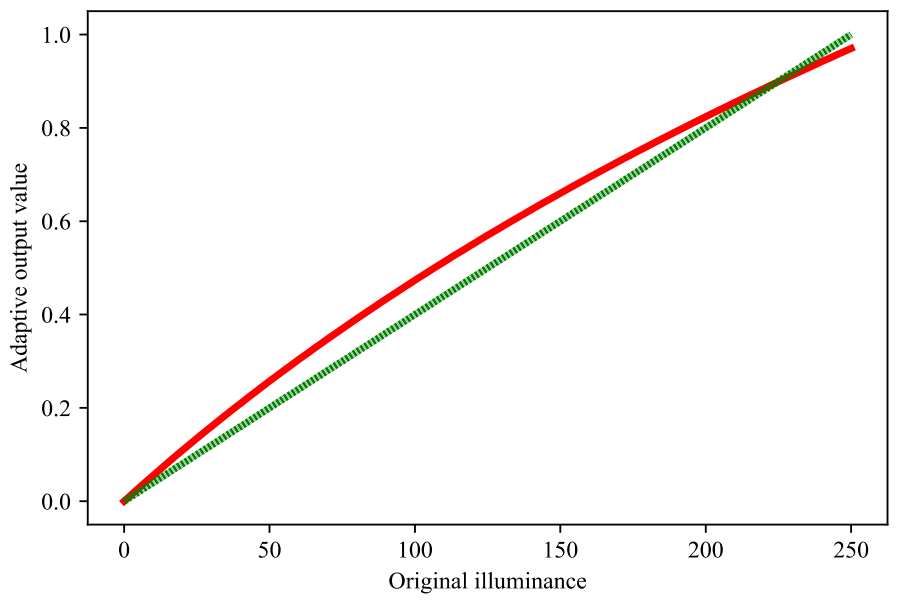

(c) Logarithmic mean 230, brightness 250

Fig. 3. Global adaptive output curve

According to the global adaptive curve, compare the corresponding relationship of the function of formula (10) in Fig. 3(a) and Fig. 3(b), when logarithmic mean is small, $L_{w}(x, y) / \overline{L_{w}}$ has a large weight, and the continuous non-linearity of the function can be approximated as a logarithmic function, so the brightness part of low illumination can be greatly improved. In contrast (b) and (c), when the maximum brightness is equal, as the logarithmic mean value goes to a high value, $L_{w}(x, y) / \overline{L_{w}}$ has a small weight, and the output curve is transformed from a log trend to a linear trend, approximating a straight line, so for high illumination images can't be improved. Therefore, it shows that adaptive enhancement will not affect high illumination images, and it will significantly enhance low illumination images.

In addition, the low illumination image in Fig. 1(a) is selected to make a global adaptive log enhancement al- 
gorithm histogram, as shown in Fig. 4(a), Fig. 4(b). It can be proved that the low illumination image is enhanced effectively.

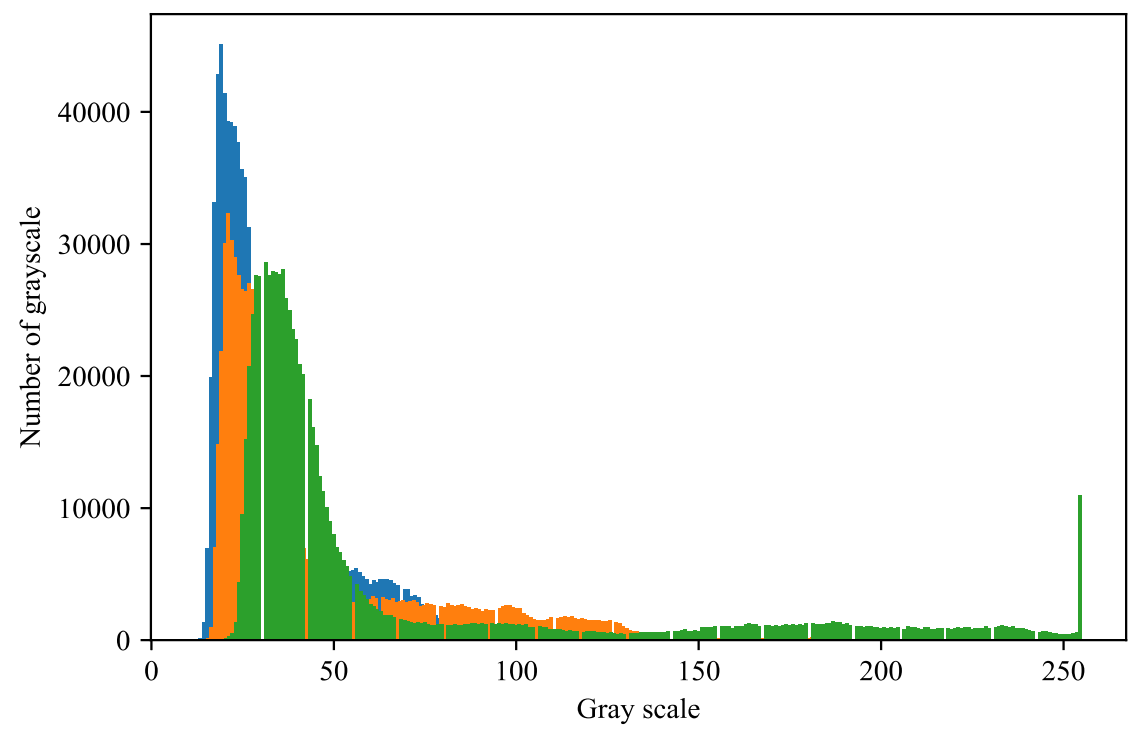

(a) Histogram of low illumination image

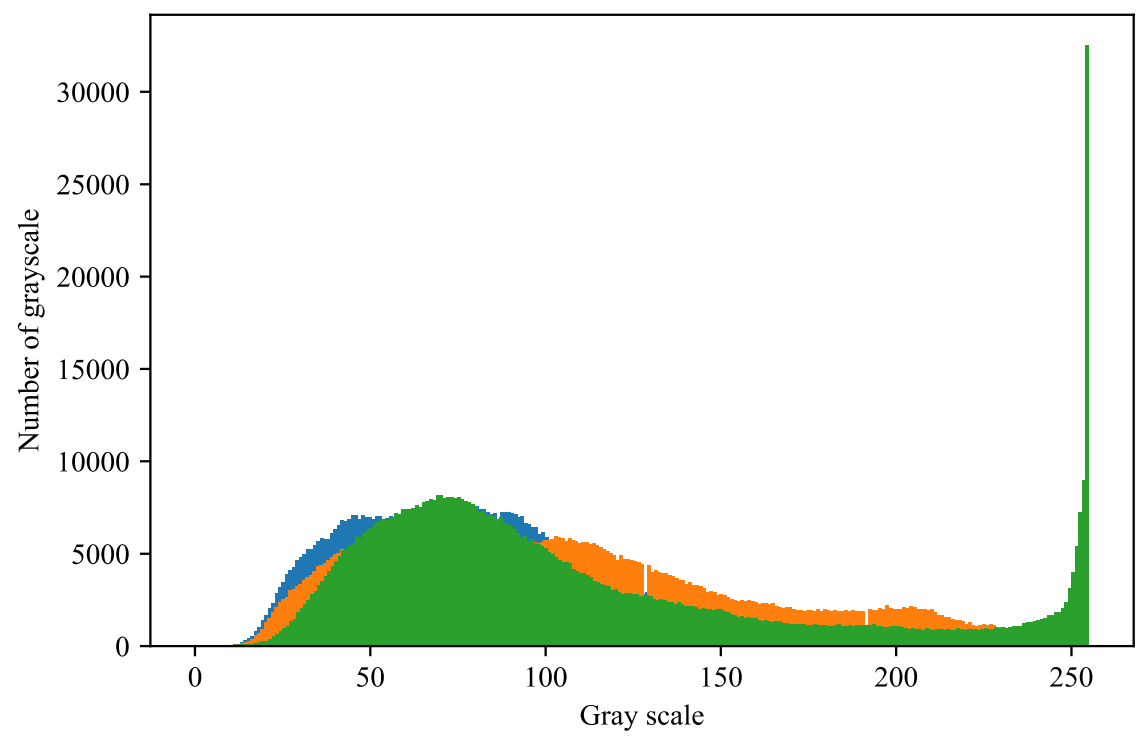

(b) Histogram of globally adaptive log-enhanced image

Fig. 4. Histogram of the image

The global adaptive logarithm enhancement algorithm is based on the logarithm property and logarithm mapping relationship, and the average brightness of the logarithm is also introduced. When the dynamic range of the scene changes, whether the image is too dark or too strong, the average brightness value is always less than or equal to the maximum brightness value, the display brightness value can be mapped to $0 \sim 1$, which ensures that the other display brightness values can be smoothly increased and no distortion will appear in the image. Therefore, for low illumination images, logarithmic mapping relationship can prevent over enhancement and will not affect high illumination images.

\section{Evaluation and Analysis of Experimental Results}

\subsection{Simulation Results}


To verify the feasibility of the algorithm, Matlab2016a was selected for experimental analysis of low illumination images. Histogram equalization, SSR, SSR with bilateral filtering, and proposed algorithm were compared. The experimental results are shown in Fig. 5.

(a)
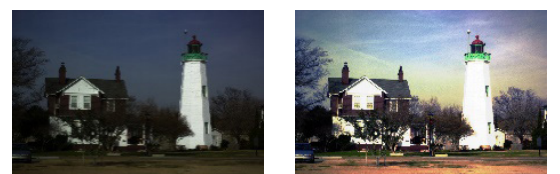

(b)

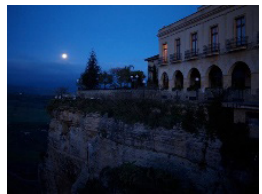

(c)

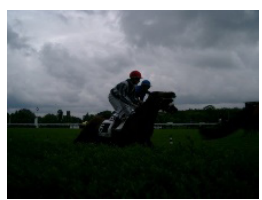

(d)

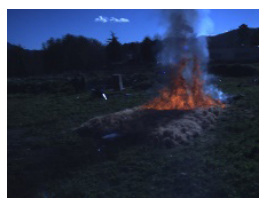

(e)

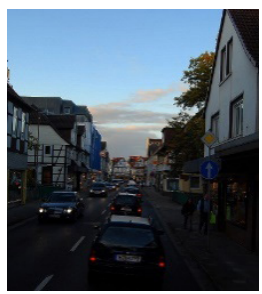

(f)

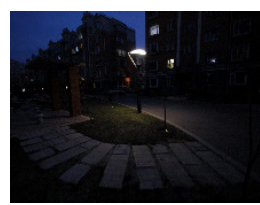

(g)

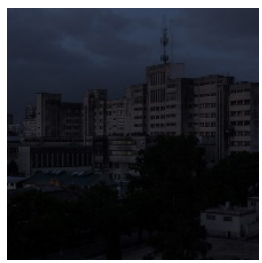

low-illumination

image
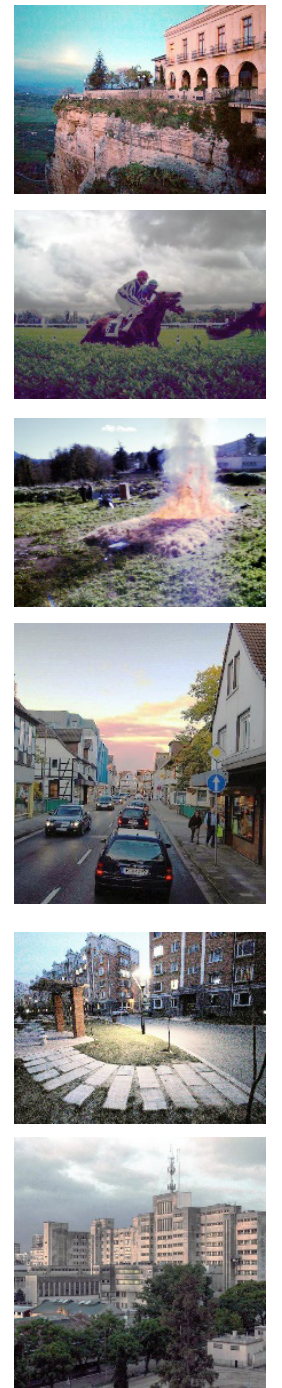

Histogram

equalized
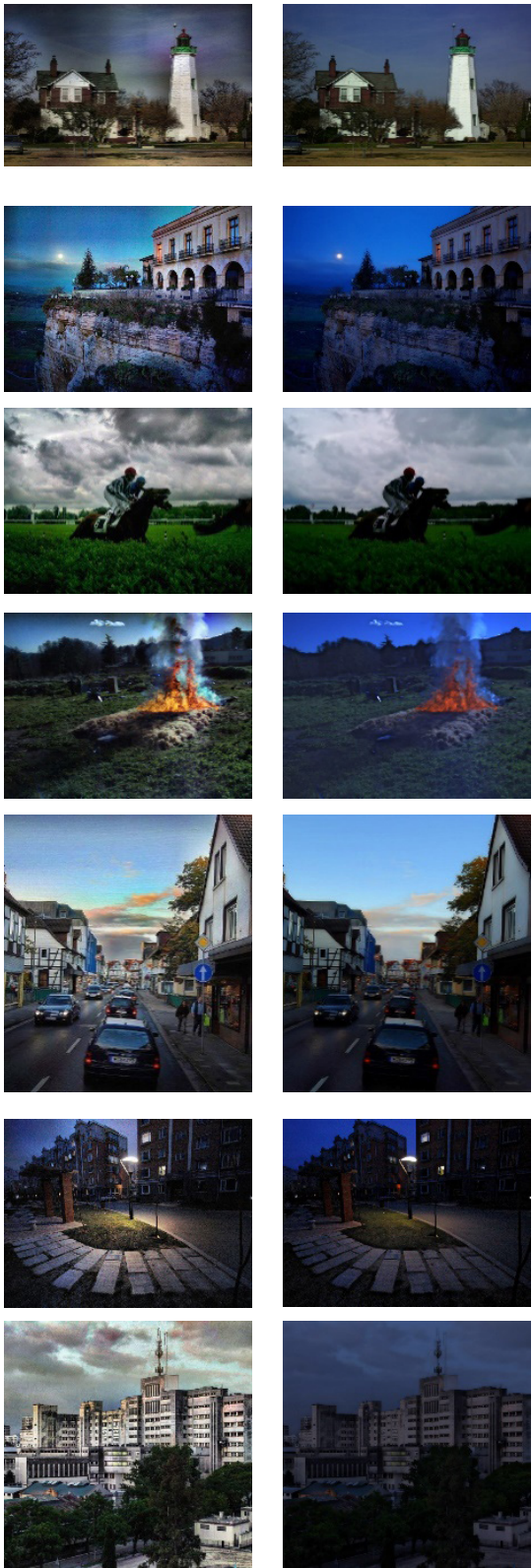

SSR
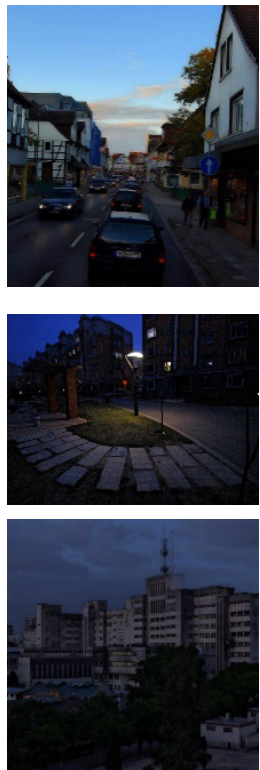

SSR with bilateral filtering
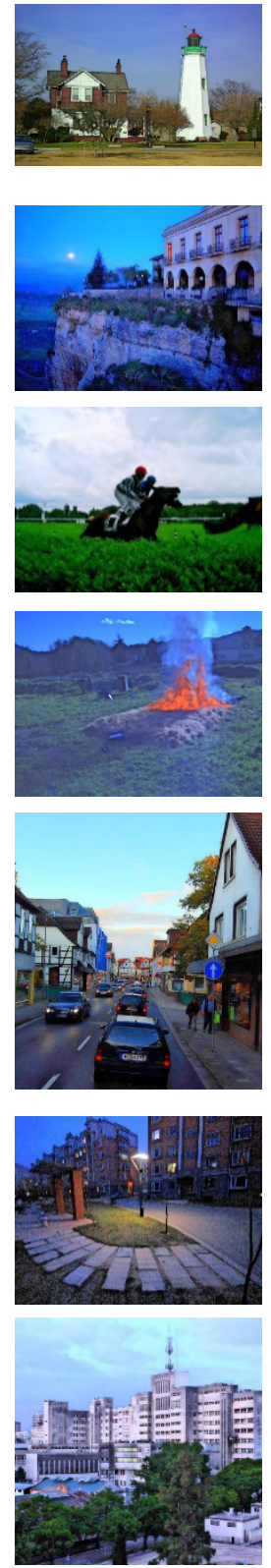

Proposed

Fig. 5. Effect of different algorithms on low-light enhancement 
In the algorithm comparison diagram, figure $\mathrm{a}, \mathrm{b}$ and $\mathrm{c}$ are dark images, figure e is uneven illumination of the original image, and figure $\mathrm{d}$, f and $\mathrm{g}$ are high-level dark images, which can hardly distinguish any detail in the image. The 7 groups of images are not clear in detail and the contrast is low. After histogram enhancement, the image appears loss of detail and over enhancement. Although SSR algorithm improves the contrast, it is still dark as a whole, and the image improvement is not obvious. SSR with bilateral filtering algorithm combine spatial weight and similar weight to make the image details more delicate, but the effect on the extremely dark images of $\mathrm{f}$ and $\mathrm{g}$ are not obvious. Compared with the other three algorithms, the proposed algorithm not only enhances the brightness of the image very well, but the details of the 7 groups images are clearer and the contrast is good, the vehicle image in figure e can see the license plate number after enhancement, figure a and figure $g$ enhancement are also obvious. This is because it introduces the global adaptive algorithm correction, and the contrast is more suitable for human subjective vision.

\subsection{Objective Evaluation of Images}

In order to further evaluate the performance of this algorithm, the average gradient, information entropy and peak signal to noise ratio are used to evaluate the image enhancement effect.

(1) Average gradient reflects image detail definition. When the average gradient of the image is larger, the image is clearer. The formula is defined as

$$
G=\frac{1}{M \times N} \sum_{i=1}^{M} \sum_{j=1}^{N} \sqrt{\frac{\left(\frac{\delta f}{\delta x}\right)^{2}+\left(\frac{\delta f}{\delta y}\right)^{2}}{2}} .
$$

Where $M \times N$ is the size of the image, $\frac{\delta f}{\delta x}$ is the gradient in the horizontal direction, and $\frac{\delta f}{\delta y}$ is the gradient in the vertical direction.

(2) Information entropy reflects the richness of image information from the perspective of information theory. In general, When the image information entropy is larger, the more abundant the information is, the formula is expressed as

$$
E=-\sum_{i=0}^{L} P(l) \log _{2} P(l)
$$

Where $P(l)$ is the probability that the gray value appears in the image, and $l$ is the gray level of the image.

(3) The peak signal-to-noise ratio is an image quality evaluation based on pixel error. When the mean value is larger, the image quality is better, the formula is expressed as

$$
P S N R=10 \log _{10} \frac{255^{2}}{\frac{1}{M \times N} \sum_{i=1}^{M} \sum_{j=1}^{N}|X(i, j)-Y(i, j)|^{2}} .
$$

$X$ is the current image, $Y$ is the original image, and $\mathrm{M}$ and $\mathrm{N}$ are the height and width of the image. The quality evaluation of different low-light image enhancement algorithms is shown in Table 1.

From the table, the proposed method is better than other algorithms in the average gradient, information entropy, mean, and peak signal-to-noise ratio, which shows that the proposed method can achieve a good enhancement effect and have an excellent visual effect. Compared with the SSR algorithm, the proposed method has stretched the image effectively, and the contrast has been enhanced. Furthermore, saturation stretching is carried out to enrich the color, and bilateral filtering is adopted to effectively avoid the halo problem, , and it can be seen that the enhanced image has richer details and obvious enhancement of edge textures, which is more in line with human visual perception. At the same time, image distortion is also avoided in the enhancement process. 
Table 1. Quality evaluation of different algorithms for low-light image enhancement

\begin{tabular}{|c|c|c|c|c|c|c|}
\hline $\begin{array}{l}\text { Test } \\
\text { picture }\end{array}$ & Evaluation & Original & $\begin{array}{c}\text { Histogram } \\
\text { equalized }\end{array}$ & SSR & $\begin{array}{c}\text { SSR with } \\
\text { bilateral filtering }\end{array}$ & Proposed \\
\hline \multirow{3}{*}{$\mathbf{a}$} & $\begin{array}{l}\text { Average } \\
\text { gradient }\end{array}$ & 0.0124 & 0.0365 & 0.0212 & 0.0322 & 0.0351 \\
\hline & $\begin{array}{c}\text { Information } \\
\text { entropy }\end{array}$ & 5.0451 & 6.7233 & 7.0914 & 6.9544 & 7.5951 \\
\hline & PSNR & 43.15 & 53.93 & 50.68 & 60.32 & 65.54 \\
\hline \multirow{3}{*}{$\mathbf{b}$} & $\begin{array}{l}\text { Average } \\
\text { gradient }\end{array}$ & 0.0214 & 0.0465 & 0.0327 & 0.0335 & 0.0366 \\
\hline & Information & 5.2421 & 5.9217 & 6.1032 & 6.4074 & 6.7454 \\
\hline & PSNR & 56.45 & 62.14 & 64.57 & 68.14 & 69.88 \\
\hline \multirow{3}{*}{ c } & $\begin{array}{c}\text { Average } \\
\text { gradient }\end{array}$ & 0.0146 & 0.0314 & 0.0344 & 0.0322 & 0.0356 \\
\hline & $\begin{array}{c}\text { Information } \\
\text { entropy }\end{array}$ & 5.5463 & 7.6225 & 6.5735 & 7.4742 & 7.6562 \\
\hline & PSNR & 46.24 & 52.12 & 48.84 & 56.43 & 58.32 \\
\hline \multirow{3}{*}{ d } & $\begin{array}{l}\text { Average } \\
\text { gradient }\end{array}$ & 0.0108 & 0.0283 & 0.0247 & 0.0266 & 0.0318 \\
\hline & $\begin{array}{c}\text { Information } \\
\text { entropy }\end{array}$ & 6.2462 & 7.4786 & 6.3434 & 7.0930 & 7.5488 \\
\hline & PSNR & 49.24 & 55.34 & 56.83 & 57.25 & 60.12 \\
\hline \multirow{3}{*}{ e } & $\begin{array}{l}\text { Average } \\
\text { gradient }\end{array}$ & 0.0213 & 0.0442 & 0.3246 & 0.3485 & 0.3768 \\
\hline & $\begin{array}{c}\text { Information } \\
\text { entropy }\end{array}$ & 6.2541 & 6.9874 & 7.0124 & 6.8544 & 7.0165 \\
\hline & PSNR & 52.14 & 58.42 & 54.37 & 53.91 & 57.48 \\
\hline \multirow{3}{*}{$\mathbf{f}$} & $\begin{array}{l}\text { Average } \\
\text { gradient }\end{array}$ & 0.0325 & 0.0378 & 0.0343 & 0.0355 & 0.0359 \\
\hline & $\begin{array}{c}\text { Information } \\
\text { entropy }\end{array}$ & 5.3647 & 5.6874 & 6.0211 & 6.1521 & 6.2245 \\
\hline & PSNR & 55.45 & 59.77 & 55.69 & 57.14 & 58.69 \\
\hline \multirow{3}{*}{$\mathbf{g}$} & $\begin{array}{l}\text { Average } \\
\text { gradient }\end{array}$ & 0.0214 & 0.0258 & 0.0231 & 0.0235 & 0.0297 \\
\hline & $\begin{array}{c}\text { Information } \\
\text { entropy }\end{array}$ & 4.4835 & 5.4343 & 4.8754 & 5.0210 & 5.3644 \\
\hline & PSNR & 43.52 & 54.21 & 52.47 & 53.63 & 54.88 \\
\hline
\end{tabular}

\section{Conclusion}

In order to improve the visual effect of low illumination images, a low illumination image enhancement technique based on the improved Retinex algorithm is proposed. Aiming at the shortcomings of the traditional Retinex algorithm, the proposed algorithm employs bilateral filtering to estimate the illumination component, which effectively prevents edge blurring and avoids the halo phenomenon. According to the problem of insufficient enhancement of image contrast, the global adaptive algorithm is adopted to correct the problem based on the characteristics of the logarithm function. Experimental results show that the proposed method can reduce the estimation error of illumination intensity and enhance the contrast, and the dark area of the low illuminance image has been significantly improved. In terms of objective evaluation, this algorithm is also better than some common low illumination enhancement algorithms. It has more details from the visual perspective, which is meaningful for the actual low illumination image preprocessing.

However, this method still has some shortcomings, such as the time-consuming problem in the enhancement process. Since this method uses MATLAB programming and has not been optimized during programming, it can be further improved by more efficient programming languages such as $\mathrm{C}++$, Python, etc.

\section{Acknowledgement}

This work (Grants 61703329) was supported by the National Natural Science Foundation of China and the National Key Research and Development Program of Shaanxi Province, China (2019KW-046).

\section{References}

[1] X.Y. Wang, H.Y. Zhang, Y.D. Wu, Y. Liu, Low-illumination image enhancement based on physical model, Journal of 
Computer Applications 35(08) 2301-2304+2310.

[2] S. Zhang, T. Wang, J. Dong, Underwater image enhancement via extended multi-scale Retinex, Neurocomputing 245(2017).

[3] D. Sen, S.K. Pal, Automatic Exact Histogram Specification for Contrast Enhancement and Visual System Based Quantitative Evaluation, IEEE Transactions on Image Processing A Publication of the IEEE Signal Processing Society 20(5)(2018) 1211-20.

[4] G. Thomas, T.D. Flores, S. Pistorius, Histogram Specification: A Fast and Flexible Method to Process Digital Images, IEEE Transactions on Instrumentation and Measurement 60(5)(2011) 1565-1578.

[5] L.L. Dong, C. Ding, W.H. Xu, Two Improved Methods Based on Histogram Equalization for Image Enhancement, Acta Electronica Sinica 46(10)(2018) 2367-2375.

[6] E. Land, J.J. McCann, Lightness and Retinex theory, Journal of the Optical Society of America 61(1)(1971) 1-11.

[7] D.J. Jobson, Z. Rahman, G.A. Woodell, Properties and performance of a center/surround Retinex, IEEE Transactions on Image Processing 6 (3)(1997) 451-462.

[8] A.B. Petro, C. Sbert, J.M. Morel, Multiscale retinex, Image Processing on Line (4)(2014) 71-88.

[9] R. Kimmel, M. Elad, D. Shaked, A Variational Framework for Retinex, International Journal of Computer Vision 52(1) (2003) 7-23.

[10]Q. Mu, Y.Y. Wei, J. Li, H.G. Li, Z.L. Li, Research on the improved Retinex algorithm for low illumination image enhancement, Journal of Harbin Engineering University 39(12)(2018) 2001-2010.

[11]Z.F. Fu, H. Zhu, Enhancement algorithms for low illumination image based on wavelet transform, Journal of Shanxi University(Natural Science Edition) 6(4)(2013) 497-504.

[12]Q.Z. Li, Q. Liu, Adaptive enhancement algorithm for low illumination images based on wavelet transform, Chinese Journal Of Lasers 42(02)(2015) 280-286.

[13]C. Li, J Guo, F. Porikli, LightenNet: A Convolutional Neural Network for weakly illuminated image enhancement, Pattern recognition letters 104(MAR.1)(2018) 15-22.

[14]H.Q. Ma, S.P. Ma, Y.L. Xu, M.M. Zhu, Low-light image enhancement based on deep convolutional neural network, Acta Optica Sinica 39(02)(2019) 99-108.

[15]J.G. Oh, M.C. Hong, Adaptive image rendering using a nonlinear mapping-function-based Retinex model, Sensors (Basel, Switzerland) 19(4)(2019).

[16]B. Biswas, P. Roy, R. Choudhuri, Microscopic image contrast and brightness enhancement using multi-scale retinex and cuckoo search algorithm, Procedia Computer Science 70(2015) 348-354.

[17]G. Papari, N. Idowu, T. Varslot, Fast bilateral filtering for denoising large 3D images, IEEE Transactions on Image Processing 26(1)(2011) 251-261.

[18]Z.C. Hao, C. Wu, H. Yang, M. Zhu, Image detail enhancement method based on multi-scale bilateral texture filter, Chinese Optics 9(4)(2016) 423-431.

[19]J.W. Dong, B. Xu, X.F. Ma, C. Han, Low-intensity image enhancement algorithm based on homomorphic filtering and multi-scale Retinex, Science Technology and Engineering 18(22)(2018) 238-242.

[20]L. Xu, Y.P. Peng, K.N. He, Research on image denoising algorithm based on improved bilateral filtering and stochastic resonance, Laser Journal 39(08)(2018) 142-148.

[21]H. Ahn, B. Keum, D. Kim, Adaptive local tone mapping based on retinex for high dynamic range images, in: Proc. IEEE International Conference on Consumer Electronics (ICCE), 2013.

[22]F. Drago, K. Myszkowski, T. Annen, Adaptive logarithmic mapping for displaying high contrast scenes, Computer Graphics Forum 22(3)(2003) 419-426. 